\title{
Aprendizaje ubicuo en estudiantes universitarios
}

\section{Ubiquitous learning in university students}

DOI: $10.46932 / \mathrm{sfjdv2n2-183}$

Received in: March 1st, 2021

Accepted in: May 30th, 2021

\author{
Mg. Javier Giraldo Huaman Camillo \\ Universidad César Vallejo \\ Correo: jhuamanc17@ucvvirtual.edu.pe \\ Dra. Mercedes María Nagamine Miyashiro \\ Universidad César Vallejo \\ Correo: mnagamine@ucv.edu.pe \\ Mg. Richard Carlos Espinoza Chávez \\ Universidad César Vallejo \\ Correo: respinozach18@ucvvirtual.edu.pe
}

\section{RESUMEN}

Se expone una revisión sobre las teorías de cómo alcanzar un aprendizaje ubicuo de matemática, para alcanzar un nuevo paradigma de aprendizaje. La conexión brindada por los dispositivos y herramientas, en especial el internet, nos ha traído nuevas posibilidades de comunicación a nivel mundial. Las TIC, han causado un cambio en la información y acceso al conocimiento, con efectos que recién estamos empezando a conocer, principalmente en el área educativa, nos brinda mayor facilidad de acceso y libertad en el aprendizaje, ya que nos permite ir a nuestro ritmo desde diferentes lugares sin la necesidad de movilizarnos a centros formativos y tampoco someternos a horarios fijos. El estudio abordo los siguientes temas: (1) la importancia de los dispositivos manuales móviles; (2) El constructivismo; (3) Cualidades del constructivismo; (4) La Conectividad de Siemens; (5) Principios de la Conectividad; (6) Ubicuidad; (7) Aprendizaje ubicuo; y finalmente (8) El aprendizaje ubicuo de matemática. Cada uno de estos temas permitió ver de una manera distinta la nueva forma de aprender. El artículo concluye mencionando la importancia del aprendizaje ubicuo para los estudiantes y profesores, en su campo laboral, personal y académico.

Palabras clave: Constructivismo, conectividad, ubicuidad, aprendizaje ubicuo, dispositivos móviles.

\begin{abstract}
A review of the theories of how to achieve ubiquitous learning in mathematics in order to achieve a new learning paradigm is presented. The connection provided by devices and tools, especially the internet, has brought us new communication possibilities worldwide. ITCs have caused a change in information and access to knowledge, with effects that we are just beginning to know, mainly in the educational area; it also provides us with greater ease of access and freedom in learning since it allows us to go at our own pace from different places without the need to move to instructive centers and not subject to fixed schedules. This study covered the following topics: (1) the importance of manual mobile devices; (2) Constructivism; (3) Constructivism Characteristics; (4) Siemens's Connectivism; (5) Connectivism Principles; (6) Ubiquity; (7) Ubiquitous Learning; and finally (8) Ubiquitous learning in mathematics. Each of these topics enabled a new point of view for learning. The article concludes by mentioning the importance of ubiquitous learning for students and teachers, in their work, personal and academic fields.
\end{abstract}


Keywords: constructivism, connectivism, ubiquity, ubiquitous learning, mobiles devices.

\section{INTRODUCCIÓN}

Hoy en día más personas aceptan que los artefactos portátiles y la permanente conectividad inalámbrica dan mayor facilidad de aprendizaje a los seres humanos, en mayor cantidad de ambiente, de forma gratis o precio pequeño. Según Burbules (2014), el aprendizaje ubicuo, es que el aprendizaje se convierte en una proposición de cualquier momento y en cualquier sitio, como resultado, los procesos de aprender están integrados más a fondo al flujo de las actividades y las comunicaciones diarias. Gutiérrez (2012), en su investigación manifiesta que, gracias a las tecnologías los alumnos pueden trabajar un sin número de actividades a la vez, además de mantener comunicación con otros miembros, pero se debe tener mucho cuidado ya que la tecnología también se puede convertir en un distractor enorme, por lo que se deben establecer actividades bien planeadas que nos ayuden a que surja un aprendizaje significativo. Por ende, el alumno se encarga de construir su aprendizaje para la cual se recomienda aplicar lo aprendido en su vida cotidiana, debe tener en cuenta la orientación de una persona que tenga un mayor conocimiento sobre un tema específico, como el profesor, además es propicio que exista una adecuada comunicación entre ellos.

Novoa, Cancino, Uribe, Garro y Mendez (2020), caracterizan el aprendizaje ubicuo en el sistema educativo en un contexto de cambios permanentes, esta necesidad permite su aprovechamiento para obtener mejores resultados en el aprendizaje considerando el uso de las herramientas tecnológicas de los estudiantes. Además, elaboran reflexiones sobre la aplicación de esta estrategia en el aula tradicional, lo que constituye un cambio de paradigma en el manejo de los tiempos, espacio y la forma de acceder a la información. En la misma línea de ideas, Hervás, Vázquez, Fernández y López (2020) manifiestan que, el descubrimiento del aprendizaje ubicuo es un hito importante para el sector educación en el siglo XXI, parte de la idea que el acceso a la información y la formación en entornos digitales ha cambiado las vidas y la forma de aprendizaje de las personas. El fácil acceso a los dispositivos digitales junto con el desarrollo de las plataformas virtuales, app y entornos digitales más dinámicos, colabora que el aprendizaje sea significativo y duradero.

La manera de aprender en estos tiempos ha cambiado, ya que el estudiante tiene la opción de aprender y adquirir conocimientos utilizando los medios tecnológicos, elegir dónde y cuándo ocurrirá el aprendizaje. Burbules (2014), en su investigación, exploró algunas de las implicancias de estos cambios, menciona que se necesita desechar la diferencia tradicional que existe entre aprendizaje formal e informal. Dicha diferencia refleja dos aspectos a tener en cuenta: primero, el contexto de aprendizaje, institucional y el otro localizado en circunstancias comunes como el domicilio. El segundo, menciona que el 
aprendizaje ubicuo le permite al ser humano aprender socialmente interactuando desde un lugar que él considere oportuno, aun cuando la persona se encuentre sola.

Pascuas y García (2020), afirman que las TIC presentan la información de manera fácil, práctica, divertida y dinámica, facilitando el contacto con muchas personas y propiciando una educación global y a distancia, pero al ser abundante e inmediata podría convertirse en un problema, pues los estudiantes limitarían su pensamiento y podrían recurrir a prácticas como el plagio, es allí que el papel del docente es de suma importancia, para estimular prácticas éticas que contribuyan al cuidado y enriquecimiento del conocimiento científico. Por otro lado, presenta las bases teóricas que permiten analizar y entender el aprendizaje ubicuo de la matemática, sus dimensiones, nos permite también conocer las competencias ubicuas que los estudiantes deben tener para el aprendizaje.

\section{DESARROLLO}

\subsection{EL CONSTRUCTIVISMO}

Según Ortiz (2015), la educación se ha visto obligada a cambiar la estructura de sus contenidos llevándolas a centrarse en los estudiantes como lo hace el constructivismo o socio cognitivo, en el cual el estudiante tiene una participación activa dentro de su aprendizaje, debido a la interacción y exploración que existe entre sus sentidos y la practica activa. Por otro lado, cuando se ha interpretado la información que se ha recibido, se puede atribuir las definiciones en cualquier lugar que se haya desarrollado la actividad, en este modelo, las personas construyen su conocimiento en la interacción con su entorno. El aprendizaje de las personas se construye una vez adquirido el conocimiento a partir de nociones previas sobre algún tema en específico, dando lugar a la elaboración de nuevos conocimientos, Hernández (2008).

Guerra (2020), manifiesta que el alumno es el actor principal al momento de construir su propio conocimiento, al cual favorece las actividades en el aula, ya que tiene un auto conocimiento estructurado. Como docente esta forma de enseñanza brinda la oportunidad de crecer, ya que estimula la actividad del pensamiento, no solo en el alumno sino también en el docente y permite conocer y aplicar las habilidades que ya traen, así como las aprendidas.

\subsection{CUALIDADES DEL CONSTRUCTIVISMO}

Para Hernández (2008), las cualidades del constructivismo se diferencia en ocho principales características: el aprendizaje representa la realidad en múltiples entornos; complejidad del mundo real; el conocimiento se construye dentro de sí mismo; identifica actividades auténticas de manera significativa dentro y fuera del contexto; el aprendizaje predeterminado se basa en situaciones reales; promover la 
reflexión en la experiencia; la construcción del conocimiento depende del contexto y la construcción del conocimiento se apoya del trabajo colaborativo.

\subsection{LA CONECTIVIDAD DE SIEMENS}

Según Siemens (2012), la conectividad permite que el aprendizaje se dé a través de la digitalización. El aprendizaje se construye atreves de la conexión entre el ser humano y la tecnología. Además, el autor explica, el efecto que la tecnología tiene sobre la manera en la que vivimos, nos comunicamos y aprendemos actualmente. Él individuo adquiere la información de una red que está constantemente retroalimentándose; es decir la nueva información deja inservible a la anterior. La habilidad para diferenciar entre una información importante y trivial es de suma importancia al igual que la capacidad de saber cuándo la información nueva adquirida altera contundentemente al conocimiento anterior. Finalmente, la conectividad permite a la persona aprender de manera digital sin importar el lugar o la circunstancia en el cual se encuentre.

La conectividad a diferencia del constructivismo, manifiesta que los aspirantes al conocimiento intentan promover el saber, brindando significados a las tareas ejecutadas, la conectividad se basa en la teoría del caos que manifiesta que el significado ya existe; por tanto, la meta del aprendiz consiste en saber organizar los modelos que aparentemente no están visibles. Según Sánchez y Costa (2019), menciona qué en la teoría del caos, no habría una estructura clara que guie al aprendiz y por lo tanto, todos podrían ser iguales o diferentes. En el Conectivismo esa red de conocimiento formada por nodos es la que estructura el aprendizaje y el conocimiento, y sirve de base de forma no determinista para futuros aprendizajes con una personalidad fluctuante, pero concreta y personal que no estaría determinada desde la teoría del caos. Por lo tanto, la habilidad para reconocer y ajustarse a los cambios es una tarea clave del aprendizaje. Para aprender en nuestra economía de conocimiento, se necesita la capacidad para hacer conexiones entre fuentes de información y en consecuencia, crear modelos de información útiles.

Además, Siemens (2006), concluye también que la conectividad está integrada por las teorías del caos, complejidad, auto organización y redes. El aprendizaje se adquiere en contextos complejos que se puede encontrar fuera de nuestro alcance, como bases de datos que se encuentra conectadas a una información especializada, el cual nos facilita aprender en los diferentes campos.

Por otro lado, Gutiérrez (2012), manifiesta que el acceso a las redes es fundamental para la conectividad, pero cabe resaltar que el acceso al internet no es igual en los diferentes países ya que un país desarrollado tiene mayores posibilidades de adquirir el conocimiento a través de la tecnología, lo cual no se da fácilmente en los países considerados como pobres, por ello la desventaja que se evidencia en la educación de sus pobladores. Además, debemos mencionar que es importante considerar la dificultad y 
desventaja a la que se enfrenta la ciudadanía que presenta algún grado de discapacidad, al hacer contacto con la tecnología. Tomando en cuenta dichas limitaciones, el autor propone considerar políticas que ayuden al acceso igualitario en las redes, para así alcanzar una educación competitiva para todas las personas.

\subsection{PRINCIPIOS DE LA CONECTIVIDAD}

El conectivismo es considerado una teoría de aprendizaje para la era digital, Siemens (2004) dispone algunos principios para la conectividad, tales como: el conocimiento al igual que el aprendizaje se encuentra, en la diversidad de opiniones. El aprendizaje consiste en conectar diferentes fuentes de información. El aprendizaje no solo se encuentra en la persona sino también fuera de ellas. El conocimiento adquirido recientemente ha alcanzado mayor importancia. El aprendizaje continuo se da a través de las conexiones permanentes entre redes, ya que ellas se encuentran en constante actualización de la información. La capacidad para observar la conexión entre ideas y conceptos es fundamental. El proceso colectivista se basa en la información actualizada.

\subsection{UBICUIDAD}

El aprendizaje ubicuo (u-learning) se conoce como el sistema de aprendizaje en línea, el cual permite que la persona logre alcanzar el conocimiento, donde y cuando él lo desee, para lo cual es necesario llevar consigo un dispositivo electrónico, Villa y Tapia (2010). Asimismo, para que el aprendizaje se adquiera de manera ubicua, es importante distribuir el espacio y tiempo, Burbules (2012). La ubicuidad hace referencia a la universalidad y a la posibilidad de desafiar las limitaciones que impone un espacio físico de manera simultánea. Por consiguiente, es flexible a la adaptación de diversos contextos en constante cambio, que a la vez permite reconocer de manera oportuna y saber cómo adaptarse a él, haciendo uso de los dispositivos móviles.

\subsection{APRENDIZAJE UBICUO}

Dentro del aprendizaje ubicuo, se encuentra un nuevo modelo educativo que es posible gracias a los medios digitales, además la abundancia de tecnología y la replicación de servicios digitales que se basan en audio y video, permiten que la formación educativa se encuentre a dispensa de las personas, sin importar el lugar o contestos siempre y cuando tenga un dispositivo, Vásquez y Sevillano (2015). Cabe resaltar que el objetivo del aprendizaje ubicuo, consiste en realizar contextos educativos generalizados o universales, en el cual se desarrolle el aprendizaje en diferentes contextos. Es decir, los alumnos una vez que hayan adquirido conocimiento tengan la oportunidad de difundir y compartir información relevante. 
Burbules (2014), manifiesta que el aprendizaje ubicuo se encuentra relacionado a tres dimensiones, en el disperso, el contenido publicado no se jerarquiza ni fragmenta y se puede difundir de manera viral; en el efímero, la información difundida es rápida y fugaz ya que circula de manera acelerada y el tercero, el importante puesto permite de manera simultánea enfocar o eliminar la información.

El modelo educativo ha cambiado, hoy en día se toma mucha importancia el móvil, por ello la ubicuidad, está compuesta por diferentes actividades formativas que se apoyan en la tecnología, algunas de las características según Carmona \& Francisco (2012): accesibilidad, todos los contenidos formativos están virtualizados y accesibles en todo momento y desde cualquier dispositivo pudiendo revisar documentos, datos o vídeos en cualquier instante. Permanente, las actividades formativas de las cuales los alumnos son los actores, se encuentra almacenada y acumulada para ser consultada en un futuro. Colaborativo, el aprendizaje se da en la relación que existe entre compañeros y profesores de manera simultánea, es decir, forma síncrona y asíncrona. Continuo, el alumno se encuentra en la capacidad de crear un hábito de aprendizaje. Natural, el uso continuo de la tecnología ha vuelto la interacción habitual, es por ello que de manera inconsciente vamos generando conocimiento, viéndose como natural. Inmediato, la información se da en un instante y en cualquier ocasión. Interacción, la persona interactúa con diferente dispositivo de manera inconsciente, también se puede dar con las diferentes herramientas de comunicación que le permite el acceso a compartir con expertos, compañeros y maestros. Adaptabilidad, se basa en la idea de adaptar los métodos de enseñanza a los estilos de aprendizaje de los alumnos; un método individualizado de enseñanza ayudará a aprender más rápido, de un modo mucho más eficaz y con un grado de comprensión elevado.

Las actividades educativas se convierten en retos y problemas que se encontraran en la vida diaria, permiten adquirir nuevos conocimientos que se encuentran presentes de forma autentica y natural (Rodríguez, 2009). Además, la U-Learning en concebido como una evolución del e-Learning y que toma la movilidad del aprendizaje m-Learning para que se dé el aprendizaje omnipresente en todo momento y lugar.

Hidalgo y Orozco (2015), presenta una imagen, la intersección del e-Learning con m-Learning y un aprendizaje móvil. 
Figura 1: Intersección entre u, e, y m de learning.

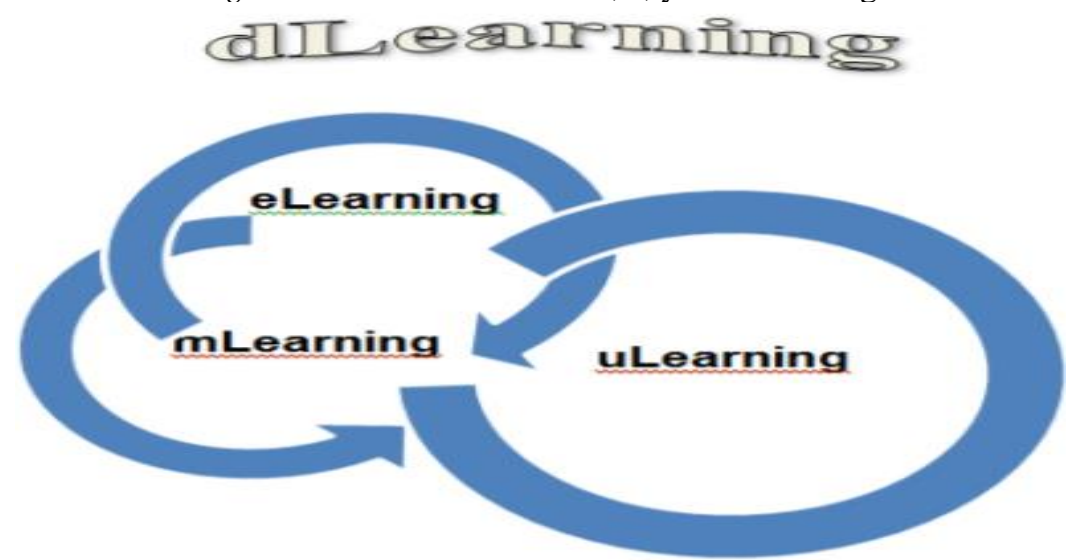

Fuente: Hidalgo y Orozco (2015)

Tabla 1: Contexto para cada tipo de aprendizaje

\begin{tabular}{cll}
\hline $\mathrm{D}$ & $\begin{array}{l}\mathrm{d}- \\
\text { Learning }\end{array}$ & $\begin{array}{l}\text { Se produce aprendizaje en cualquier } \\
\text { momento y lugar }\end{array}$ \\
\hline $\mathrm{E}$ & $\begin{array}{l}\mathrm{e}- \\
\text { Learning }\end{array}$ & Dispositivos fijos \\
\hline $\mathrm{M}$ & $\begin{array}{l}\text { m- } \\
\text { Learning }\end{array}$ & Dispositivos móviles \\
\hline $\mathrm{U}$ & $\begin{array}{l}\text { u- } \\
\text { Learning }\end{array}$ & Dispositivos fijos y móviles \\
\hline
\end{tabular}

Fuente: Elaboracion propia

Asimismo, Hidalgo y Orozco (2015), manifiesta que el u-Learning se da más como un aprendizaje informal, por tal razón es importante que para el aprendizaje formal se desarrollen actividades enfocadas al u-Learning y para cualquier contexto de tal forma que el alumno siempre estará rodeado de varios elementos. 
Figura 2: Elemento de los que se rodea un alumno en el aprendizaje u-Learning.

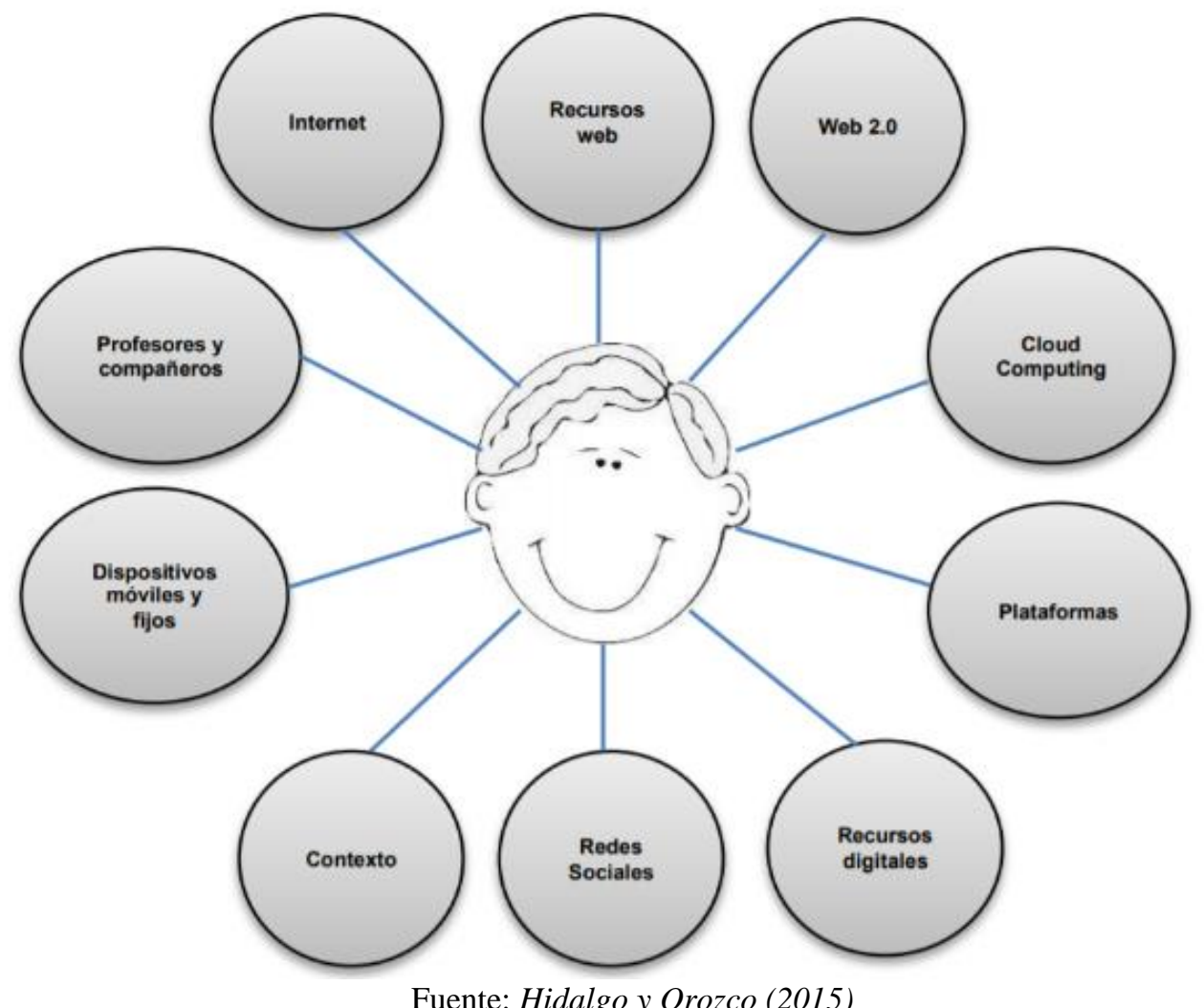

Fuente: Hidalgo y Orozco (2015)

\subsection{EL APRENDIZAJE UBICUO DE MATEMÁTICA}

Según Humanante (2016), manifiesta que en el ámbito de las universidades el aprendizaje ubicuo surge como una opción de solventar las deficiencias que existe en el aprendizaje institucional, además la ubicuidad potencia el aprendizaje de la información a través de la tecnología, facilitando así el aprendizaje ubicuo de matemática. Es por ello los alumnos utilizan dichos entornos para reforzar la parte teórica y práctica en el curso de matemática, y así puedan resolver las actividades dejadas por los docentes, mejorando así su aprendizaje.

\section{CONCLUSIONES}

Se ha presentado la importancia del aprendizaje ubicuo, brindada por los informes que provienen de los diferentes artículos que se ha revisado. El propósito fue dar mayor conocimiento sobre la ubicuidad, que se respalda en la tecnología de la información y comunicación, que tiene como principales recursos las redes sociales y el internet, en la cual las personas tienen libre acceso a los diferentes contenidos de aprendizaje, que les brinda un beneficio laboral y personal sin tener limitación alguna, solo hace falta tener la motivación de aprender.

La ubicuidad del aprendizaje debe ser un paradigma a seguir para los docentes de hoy en día, ya que les impulsa a conocer nuevas formas y maneras de enseñar, a la vez los alumnos logren adquirir 
conocimientos relevantes, ya que a través del aprendizaje ubicuo, tienen nuevas posibilidades de aprender sin tener que estar físicamente sentado en un escritorio, además de las ventajas que nos proporciona la tecnología móvil, con la cual podemos tener acceso al Internet sin la necesidad de tener un cable para conectarse.

La importancia del aprendizaje ubicuo en la matemática, en el proceso de aprendizaje, no se debe únicamente al libre acceso de información almacenada, sino también al conocimiento que se genera en la interacción con la red, para poder comprender y explicar este nuevo fenómeno que se está dando en el ámbito educativo, por ello es necesario hacer énfasis en las teorías anteriormente presentadas.

En posteriores investigaciones se recomienda que la ubicuidad debe ser investigada, con la finalidad de seguir ampliando el conocimiento y el aprendizaje adquirido por las nuevas tecnologías, así fortalecer las teorías existentes, de esta forma fortalecer las competencias ubicuas, para que el estudiante pueda tener un aprendizaje deseado. También debemos tener en cuenta que al igual que algunos lugares del mundo, en el Perú no todos los estudiantes tienen acceso al internet, por ello el Estado peruano debe contribuir con aquellos alumnos que no tienen acceso a dichos recursos y así eliminar las brechas sociales. 


\section{REFERENCIAS}

Burbules, N. (2012). "Ubiquitous Learning and the Future of Teaching". En Revista Encounters on Education, Vol. 13, 2012, 3 - 14, ISSN 1925-8992. Disponible en: https://dialnet.unirioja.es/servlet/articulo?codigo=4100463\&orden=371206\&info=link

Burbules, N. (2012) Aprendizaje Ubicuo, entrevista realizada por IIPEE - UNESCO. Video disponibleen: http://www.webinar.org.ar/conferencias/entrevista-nicholas-burbules

Burbules, N. (2014). "Los significados de aprendizaje ubicuo”. En Revista Redalyc, Volumen 22, Número 104, ISSN 1068-2341. Disponible en: https://www.redalyc.org/pdf/2750/275031898105.pdf

Burbules, N. (2014). "El aprendizaje ubicuo: nuevos contextos, nuevos procesos”. En Revista Educación y Sociedad. p. 131-135. Disponible https://fh.mdp.edu.ar/revistas/index.php/entramados/article/view/1084/1127

Carmona, L. \& Francisco, P. (2012). "U-Learning: La revolución del aprendizaje”. Recuperado el 02 de Mayo de 2015, de Area de Talent \& Organization de Accenture. Disponible en: www.accenture.es/topinformes

Guerra, J. (2020). "El constructivismo en la educación y el aporte de la teoría sociocultural de Vygotsky para comprender la construcción del conocimiento en el ser humano". En Revista Dilemas Contemporáneos: Educación, Política y Valores, DOI:10.46377/dilemas.v32i1.2033. Año: VII Número: 2 Artículo no.:77. Disponible en: https://www.researchgate.net/publication/338402805

Gutiérrez, L. (2012). "Conectivismo como teoría de aprendizaje: conceptos, ideas, y posibles limitaciones". En Revista educación y tecnología, págs. 111-122. Disponible en: http://revistas.umce.cl/index.php/edytec/article/view/39/pdf

Hervás C, Vázquez E, Fernández, J, López, E (2020). “Innovación e Investigación Sobre El Aprendizaje Ubicuo y Móvil En La Educación Superior". Ediciones Octaedro; http://search.ebscohost.com/login.aspx?direct=true \&db=edsebk\&AN=2169418\&lang=es\&site=eds-live

Hernández, S. (2008). "El modelo constructivista con las nuevas tecnologías: aplicado en el proceso de aprendizaje". En Revista de universidad y Sociedad del Conocimiento, RUSC vol. 5 n. ${ }^{\circ} 2$ (2008) | ISSN 1698-580x. Disponible en: https://www.raco.cat/index.php/RUSC/article/viewFile/253968/340755

Hidalgo, S. y Orozco, M. (2015). "Trabajando con Aprendizaje Ubicuo en los alumnos que cursan la materia de Tecnologías de la Información”. En Revista Ride, ISSN 2007 - 7467. Vol. 6, Núm. 11 Julio Diciembre 2015. Disponible en: https://www.redalyc.org/pdf/4981/498150319036.pdf

Humanante, P. (2016). "Entornos Personales de Aprendizaje Móvil (MPLE) en la Educación Superior". Tesis doctoral, Disponible en: https://repositorio.grial.eu/handle/grial/635

Novoa, P, Cancino, R, Uribe Y, Garro L y Mendez G. (2020) "El Aprendizaje Ubicuo En El Proceso de Enseñanza Aprendizaje".

UNAN-Managua; http://search.ebscohost.com/login.aspx?direct=true\&db=edsbas\&AN=edsbas.EF9FC7A1\&lang=es\&site =eds-live 
Ortiz, D. (2015). "El constructivismo como teoría y método de enseñanza". En Revista Redalyc, DOI: 10.17163/soph.n19.2015.04, Sophia: colección de Filosofía de la Educación, 19 (2), pp. 93110.Disponible en: https://www.redalyc.org/pdf/4418/441846096005.pdf

Pascua, S y García, J. (2020). “Dispositivos móviles en la educación: Tendencias e impacto para la innovación". En Revista politécnica, ISSN 1900-2351 (Impreso), ISSN 2256-5353 (En línea), Año 16, Número 31, páginas 97-109.Disponible en: https://revistas.elpoli.edu.co/index.php/pol/article/view/1702/1432

Rodríguez, S. (2009). "Informática ubicua y aprendizaje ubicuo". Recuperado el 10 de Mayo de 2015, de Cajón de Sastre. Disponible en: http://recursostic.educacion.es/observatorio/web/fr/cajon-de-sastre/38cajon-desastre/910-monografico-informatica-ubicua-y-aprendizaje-ubicuo?format=pdf

Sánchez, R. y Costa, O. (2019). "Orígenes del conectivismo como nuevo paradigma del aprendizaje en la era digital". En Revista Educación y Humanismo, ISSN: 0124-2121. E-ISSN: 2665-2420.DOI: http://dx10.17081/eduhum.21.36.3265.

Disponible en:

https://dialnet.unirioja.es/servlet/articulo?codigo $=6786548$

Siemens, G. (2004). "Connectivism: A learning theory for the digital age". International journal of instructional technology and distance learning. Disponible en:https://www.comenius.cl/recursos/virtual/minsal_v2/Modulo_1/Recursos/Lectura/conectivismo_Sie mens.pdf

Siemens, G. (2006). Knowing knowledge. Morrisville, Estados Unidos: ISBN: 978-90-815937-1-7. Una versión con licencia Creative Commons de esta obra está disponible en: https://app.box.com/s/31mg21z77d

Siemens, G. (2012). Conferencia completa en español de su teoría del conectivismo en el marco del Encuentro Internacional de Educación 2012 - 2013 de la Fundación Telefónica realizado en la ciudad de Lima, Perú. Video disponible en: https://www.youtube.com/watch?v=s77NwWkVth8\&feature=emb_rel_pause

Vásquez, E. y Sevillano, M. (2015). “Dispositivos digitales móviles en Educación: El aprendizaje ubicuo”. ISBN libro papel: 978-84-277-2100-5, impreso en España. Imprime: Lavel. pol. ind. los llanos.28970 humanes (Madrid). Disponible en: https://books.google.es/books?id=C8fDCQAAQBAJ\&lpg=PA9\&ots=1zTXSEyPme\&dq=aprendizaje $\%$ 20ubicuos\&lr\&hl=es\&pg=PA9\#v=onepage \&q=aprendizaje\%20ubicuos\&f=false

Villa, M y tapia, F (2010). “aprendizaje ubicuo en la enseñanza de las matemáticas”. En Revista Estudios Culturales, ISSN-e 1856-8769, No. 5, 2010, págs. 123-136 Disponible. En: http://dialnet.unirioja.es/servlet/articulo?codigo=3739983 